\title{
Matter-wave localization in a weakly perturbed optical lattice
}

\author{
Yongshan Cheng ${ }^{1, *}$ and S. K. Adhikari ${ }^{2, \dagger}$ \\ ${ }^{1}$ Department of Physics, Hubei Normal University, Huangshi 435002, People's Republic of China \\ ${ }^{2}$ Instituto de Física Teórica, Universidade Estadual Paulista, 01.140-070 São Paulo, São Paulo, Brazil
}

(Received 3 September 2011; published 29 November 2011)

\begin{abstract}
By numerical solution and variational approximation of the Gross-Pitaevskii equation, we studied the localization of a noninteracting and weakly interacting Bose-Einstein condensate in a weakly perturbed optical lattice in one and three dimensions. The perturbation achieved through a weak delocalizing expulsive or a linear potential as well as a weak localizing harmonic potential removes the periodicity of the optical lattice and leads to localization. We also studied some dynamics of the localized state confirming its stability.
\end{abstract}

DOI: 10.1103/PhysRevA.84.053634

PACS number(s): 03.75.Nt, 64.60.Cn

\section{INTRODUCTION}

Anderson suggested the possibility of localization of an electron wave in a nonperiodic random potential about 50 years ago [1]. After the recent experimental realization of Anderson localization of a Bose-Einstein condensate (BEC) [2,3], this topic has been the subject of intense research activities. Billy et al. [2] observed the exponential tail of the spatial density distribution of a ${ }^{87} \mathrm{Rb}$ BEC after releasing it into a one-dimensional (1D) waveguide with a controlled disorder potential created by a laser speckle. Roati et al. [3] observed the Anderson localization of a noninteracting ${ }^{39} \mathrm{~K}$ BEC in a 1D quasiperiodic bichromatic optical lattice (OL). Recently, the experimental realizations of three-dimensional (3D) localization of ultracold gases of ${ }^{40} \mathrm{~K}$ [4] and ${ }^{87} \mathrm{Rb}$ [5] atoms in a $3 \mathrm{D}$ speckle potential were also reported. In the experimental studies of localization of a BEC, bichromatic OL [3,6], shaken OL [7], cold-atom lattice [8], and speckle potentials [2] have been used. There have also been studies of the effect of a repulsive atomic interaction on localization [9]. Bichromatic OL [10-12], a cold-atom lattice [13,14], random potentials [15-18], and OL plus a harmonic trap [19], among others, have been used in theoretical studies. Quasiperiodic [19,20] or random [21-24] potentials for Anderson localization were realized by optical means. In Ref. [19], localization and Bloch oscillation of a BEC were studied in two spatial dimensions for OL perturbed by a harmonic potential [25]. Here we shall consider localization in $1 \mathrm{D}$ and 3D in the same potential.

However, there are other ways of obtaining a nonperiodic OL. Here we consider another approach for perturbing the OL, so that the periodicity of the original OL is completely removed. If we perturb the OL by a weakly delocalizing (i) linear potential or (ii) expulsive harmonic potential, the periodicity of the OL is removed without adding an extra confining term to the OL. Both linear [26,27] and expulsive [28] harmonic potentials can be made in the laboratory by optical and magnetic means. In Ref. [27] a tilted trap was used for the observation of Bloch oscillations in a repulsive BEC, where the external potential is simply given by the sum of the linear (gravitational) component and the OL laser field. In

\footnotetext{
*yong_shan@163.com

†adhikari44@yahoo.com; http://www.ift.unesp.br/users/adhikari
}

Ref. [28] an expulsive potential was used to study solitons in BECs.

Here, with the numerical solution of the Gross-Pitaevskii (GP) equation, we investigate the localization of cigar-shaped BECs in a 1D OL, weakly perturbed by linear and expulsive confining harmonic potentials. We also considered adding a weak confining harmonic trap to the OL, which removes the periodicity of the OL to obtain localization. The localized states so obtained are completely distinct and had a different spatial extension from the states trapped in the confining harmonic trap alone and hence were localized due to the nonperiodic OL. It is found that the localized states in the three types of periodicity-broken OL are practically the same and do not depend on the weak perturbation used to remove the periodicity of the OL. In most cases the density profile of the central part of the localized state is quite similar to a Gaussian shape. Hence it is worthwhile to solve the GP equation analytically using a Gaussian variational ansatz. Indeed, we find that the variational solution fits well the density distribution in the central region, especially when the localization takes place essentially on a single site of the perturbed OL. However, the localized states usually have a long undulating exponentially decaying tail in either direction and extend to several sites of the OL corresponding to Anderson localization in a weak potential $[15,24]$. The stability of the localized state under small perturbation is also investigated.

We also extend our study to localization in 3D. In 3D, the effect of disorder on an initial state at large times depends strongly on the energy, and a mobility edge was predicted, which corresponds to a critical energy at which a transition from localized to extended character of the state occurs [29]. As observed in the recent experiments in three dimensions [4,5], a two-component density distribution emerges at large times consisting of an expanding mobile component and a nondiffusing localized component after the ultracold gas of atoms are released into an optical speckle field. In this paper, different from the experimental scenario, we focus our attention on the stationary localized states and not on the large-time expansion dynamics. We find that the weakly perturbed 3D OL also leads to localization. In the 3D realm, we only present results for the expulsive perturbation of the OL, as other perturbations lead to similar results. In this case we show results for noninteracting BECs as well as for weakly repulsive BECs. The numerical chemical potentials are in 
good agreement with the variational results in all cases. The localized states of the 3D BEC are found to be stable against small perturbations.

In Sec. II we present the 1D and 3D models as well as their variational analysis used in this study of localization. In Sec. III we study numerically the statics and dynamics of a 1D and a 3D BEC by analyzing the atom density profiles and chemical potential of the localized states in a perturbed OL and compare the numerical results with the variational solutions. The study of a sustained small oscillation of the localized states upon external perturbation confirmed their stability. In Sec. IV we present a summary of our findings.

\section{ANALYTICAL CONSIDERATION}

The dynamic behavior of a weakly interacting Bose gas at low temperature on a 3D OL is well described by the GP equation, which has the form of a $(3+1)$-dimensional nonlinear Schrödinger equation [30]:

$$
\begin{gathered}
i \frac{\partial \Phi}{\partial t}=-\frac{1}{2} \nabla^{2} \Phi+G|\Phi|^{2} \Phi+U(x, y, z) \Phi, \\
U(x, y, z)=-V_{0}[\cos (2 x)+\cos (2 y)+\cos (2 z)],
\end{gathered}
$$

where $\Phi \equiv \Phi(x, y, z, t)$ is the BEC wave function with normalization $\iiint d x d y d z|\Phi|^{2}=1$ and $\nabla^{2}$ is the 3D Laplacian. The constant $G \equiv 4 \pi a N$ characterizes the two-body interaction, $N$ is the total number of atoms, $a$ is the atomic scattering length, and $U$ is the 3D OL. The spatial variable, time, density $|\Phi(x, y, z, t)|^{2}$, and potential are measured in units of $l_{0}=\lambda /(2 \pi), m l_{0}^{2} / \hbar, l_{0}^{-3}$, and $2 E_{R}$, respectively, where $E_{R}=h^{2} /\left(2 m \lambda^{2}\right)$ is the recoil energy of an atom absorbing a lattice photon, $\lambda$ is the wavelength of the OL potential, and $m$ is the atomic mass.

We consider a 3D trapping potential $U(x, y, z)$ made up of three weakly perturbed OL components defined as

$$
U(x, y, z)=\sum_{i=1}^{3} V\left(x_{i}\right)
$$

where $x_{1,2,3}$ correspond to $x, y, z$, respectively. Each of the three components has the following forms:

$$
\begin{gathered}
V\left(x_{i}\right)=-V_{0} \cos \left(2 x_{i}\right)-C x_{i}, \\
V\left(x_{i}\right)=-V_{0} \cos \left(2 x_{i}\right) \pm C x_{i}^{2},
\end{gathered}
$$

with $V_{0}=5$, corresponding to perturbations by a weak linear [Eq. (4)] and confining [Eq. (5), positive sign] and expulsive [Eq. (5), negative sign] traps, with strength $C$. These perturbations slightly modify the periodic OL and generate nonperiodic OL potentials.

We also consider a cigar-shaped condensate oriented in the longitudinal direction ( $x$ direction). The trapping potential is a weakly perturbed OL in the longitudinal direction, and the transverse $(y, z$ directions) dynamics of the condensate is frozen to the respective ground states of harmonic traps. This cigar-shaped system will be described by using an effective 1D model derived from the 3D GP equation (1) by integrating over the transverse variables [31]. Then, the time-dependent

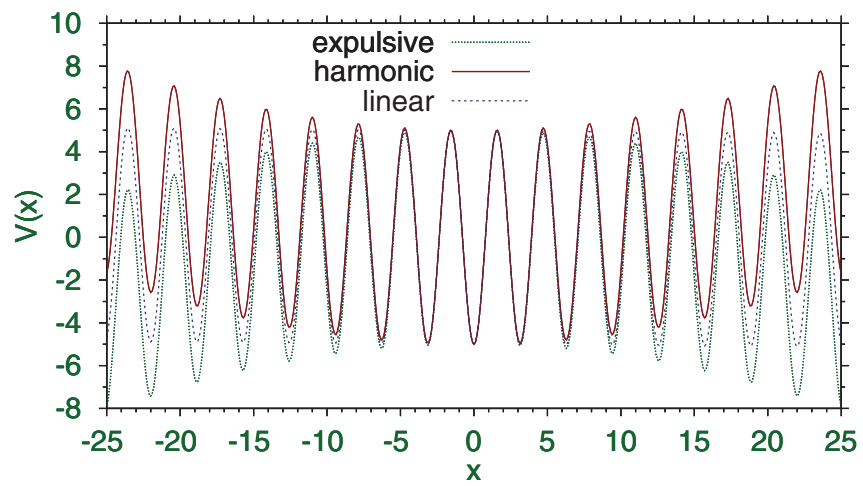

FIG. 1. (Color online) The linear, confining, and expulsive harmonic potentials $V(x)$ as a function of $x$ in dimensionless units from Eqs. (4) and (5) for $C=0.005, V_{0}=5$.

wave function $\phi \equiv \phi(x, t)$ of a BEC can be described by the following $1 \mathrm{D}$ GP equation $[31,32]$ :

$$
i \frac{\partial \phi}{\partial t}=-\frac{1}{2} \frac{\partial^{2} \phi}{\partial x^{2}}+g|\phi|^{2} \phi+V(x) \phi,
$$

with normalization $\int_{-\infty}^{\infty}|\phi|^{2} d x=1$, nonlinearity $g=$ $2 a N / a_{\perp}^{2}$, and potential $V(x)$ given by Eq. (4) or (5). The spatial variable $x$, time $t$, density $|\phi|^{2}$, and energy are expressed in normalized units $a_{\perp}=\sqrt{\hbar /\left(m \omega_{\perp}\right)}, \omega_{\perp}^{-1}, a_{\perp}^{-1}$, and $\hbar \omega_{\perp}$, where $\omega_{\perp}$ is the angular frequency of the transverse trap. These weakly perturbed OLs along the $x$ axis are illustrated in Fig. 1, which shows that the OL dominates for small $x$, but the periodicity of the original OL is completely removed.

Stationary solutions $u(x, y, z)$ to Eq. (1) can be given by $\Phi(x, y, z, t)=\exp (-i \mu t) u(x, y, z)$, with $\mu$ being the chemical potential. For convenience, we use unified notation $u$ to denote the real wave function with $u \equiv u(x)$ for $1 \mathrm{D}$ and $u \equiv u(x, y, z)$ for 3D BECs, respectively. We choose $d(=1,3)$ to denote the dimension of space. The stationary real wave function obeys

$$
\mu u=-\frac{1}{2} \sum_{i=1}^{d} \frac{\partial^{2} u}{\partial x_{i}^{2}}+G u^{3}+\sum_{i=1}^{d} V\left(x_{i}\right) u .
$$

The nonlinearity $G$ should be replaced by $g$ in one dimension. Equation (7) can be derived from the Lagrangian density

$$
\mathcal{L}=\mu u^{2}-\frac{1}{2} \sum_{i=1}^{d}\left(\frac{\partial u}{\partial x_{i}}\right)^{2}-G \frac{u^{4}}{2}-\sum_{i=1}^{d} V\left(x_{i}\right) u^{2} .
$$

To apply the variational approximation to Eq. (7), we use the variational Gaussian ansatz:

$$
u=\left(\frac{1}{\pi^{1 / 4} \sqrt{w}}\right)^{d} \sqrt{\mathcal{N}} \prod_{i=1}^{d} \exp \left(-\frac{x_{i}^{2}}{2 w^{2}}\right),
$$

where $w$ is the width and $\mathcal{N}$ is the normalization of the localized BEC. The effective Lagrangian of the system can be found upon by substituting Eq. (9) into Eq. (8) and integrating over space [33]:

$$
\begin{aligned}
L= & \mu \mathcal{N}-\mu-\frac{d \mathcal{N}}{4 w^{2}}-\frac{G \mathcal{N}^{2}}{2}\left(\frac{1}{\sqrt{2 \pi} w}\right)^{d}+d V_{0} \mathcal{N} e^{-w^{2}} \\
& -R d \frac{\mathcal{N} w^{2}}{2}
\end{aligned}
$$


where $R=0$ for the linear potential and $R= \pm C$ for the confining and expulsive harmonic potential. The Euler-Lagrange equation $\partial L / \partial \mu=0$ yields the normalization $\mathcal{N}=1$. We use it in the following equations. The remaining equations $\partial L / \partial w=\partial L / \partial \mathcal{N}=0$ yield, respectively,

$$
\begin{aligned}
& 1=-\frac{G}{(2 \pi)^{d / 2} w^{d-2}}+4 V_{0} w^{4} \exp \left(-w^{2}\right)+2 R w^{4}, \\
& \mu=\frac{d}{4 w^{2}}+\frac{G}{(w \sqrt{2 \pi})^{d}}-d V_{0} \exp \left(-w^{2}\right)+d R \frac{w^{2}}{2},
\end{aligned}
$$

and determine the width $w$ and the chemical potential $\mu$. The linear perturbation has no effect on the width and chemical potential of the stationary localized states because $R=0$ for the linear perturbation.

\section{NUMERICAL RESULTS}

We performed the numerical integration of GP equations (1) and (6) employing the imaginary or real-time split-step Fourier spectral method with a space step of 0.04 and a time step of 0.001 . We checked the accuracy of the results by varying the space and time steps and the total number of space and time steps. We also checked the results with those obtained from a split-step Crank-Nicolson algorithm using same steps [34].

By numerically solving equation (6), we first investigate the effects of the weakly perturbed OL on a localized state in a 1D BEC. To obtain a stationary localized state, the initial input pulse is taken as $\phi(x, 0)=\exp \left(-x^{2} / 2\right) / \pi^{1 / 4}$ with a parabolic trap $V^{\prime}(x)=x^{2} / 2$ in our numerical integration. During the numerical simulation, the parabolic trap is slowly turned off, and the potential (4) or (5) and the nonlinearity $g$ is slowly turned on with a very small increment $(=0.00001)$ in each time step. The time evolution is continued until the parabolic trap is completely turned off and the potential (4) or (5) is completely turned on and the the nonlinearity $g$ has the desired value.

In Fig. 2 we exhibit typical numerical and variational results of the localized states with different perturbations for $g=0$. In the case of the linearly perturbed OL [Eq. (4)], Fig. 2(a) shows that the numerical density profiles of the central part of the localized states are the same for $C=0.005,0.05$, and 0.1 and the numerical chemical potentials are all -2.897 , in agreement with the variational result of -2.894 . For confining and expulsive perturbed OL (5), in Fig. 2(b) we
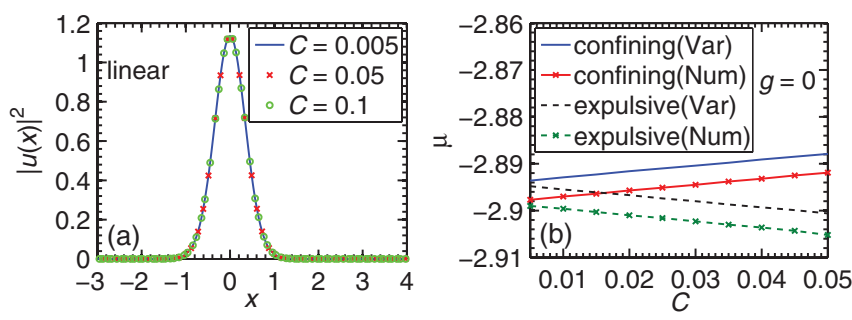

FIG. 2. (Color online) (a) Normalized numerical density profile $|u(x)|^{2}$ of the localized BEC versus normalized $x$ for potential (4) with $C=0.1,0.05$, and 0.005 and $g=0$. (b) Normalized numerical (Num) and variational (Var) chemical potentials $\mu$ of the localized BEC with $g=0$ vs $C$ for the confining and expulsive perturbed OL (5). plot the numerical and variational chemical potentials $\mu$ of the localized states. In the case of the expulsive perturbation, the chemical potential decreases with the perturbation strength. The opposite happens for the confining perturbation. The reason is that an expulsive perturbation weakens the trapping of the OL and a confining perturbation strengthens the trapping. For small $C$, the influence of $C$ on the density profile and chemical potential of a localized state is small. We also find that the stability of the localized states is related to the perturbation strength $C$. If we increase $C$, the stability situation does not improve. But if we reduce $C$ the localized states could be unstable as $C \rightarrow 0$. So we keep $C \geqslant 0.005$ in this paper.

With the periodic potential $-5 \cos (2 x)$, the noninteracting Schrödinger equation [viz., $g=0$ in Eq. (6)] permits only delocalized states in the form of Bloch waves. Localization is possible in the noninteracting Schrödinger equation due to the nonperiodic nature of the perturbed OL, as shown in Figs. 3(a) and 3(c), where we exhibit the typical density profiles of the localized states in the linear and confining harmonic potentials for $g=0$ and 10 and compare them with the corresponding variational results. To confirm that these localized states are induced by the weak perturbation of the OL, in Fig. 3(c), we also show the localized state for $g=0$ for the confining harmonic potential alone (see the black dot-dashed line). This state has a different spatial extension and can be distinguished from the other states localized due to the perturbation of the OL. Figures 3(b) and 3(d) show, respectively, the effect of the nonlinearity on the chemical potential (again in agreement with the prediction of the variational results). The corresponding results for the expulsive perturbed potential are in agreement with those of the confining potential and are not shown here. To understand the effects of $g$ on $\mu$ and on localization in general, it is useful to consider the variational equations (11) and (12);
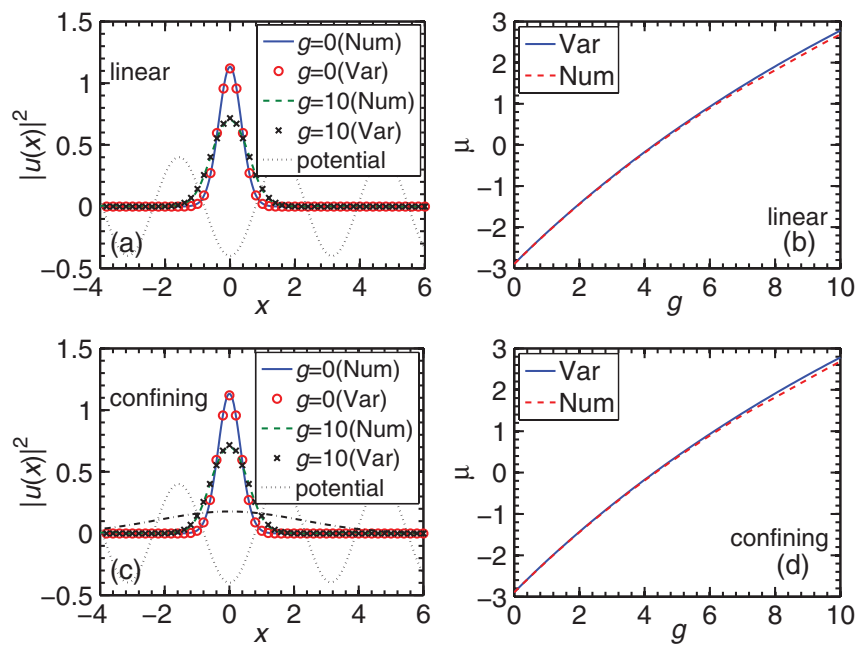

FIG. 3. (Color online) Numerical (Num) and variational (Var) density profiles $|u(x)|^{2}$ of the localized BEC vs $x$ for the (a) linear and (c) confining harmonic perturbations of the OL with $C=0.005$ and $g=0$ and 10 , respectively. In (c), we also show the density profile of the localized BEC trapped by only the confining harmonic potential $V(x)=0.005 x^{2}$ with $g=0$ (the black dot-dashed line). The numerical (Num) and variational (Var) chemical potentials $\mu$ of the localized BEC vs $g$ for (b) linear and (d) confining perturbations of OL. All quantities are dimensionless. 
for $g=0, \mu=-2.894$. As $g$ is increased, $\mu$ increases, and the system is more repulsive and extends over more region in space and the localization length increases. Eventually, at $g \approx 18.6$ the state is completely delocalized, and beyond this value of $g$, no localization is possible, and there is no real solution of Eq. (11) for the width.

As a typical Hamiltonian system, the stability of an ordinary localized BEC can be determined by the Vakhitov-Kolokolov (VK) criterion [35]. Of course, the direct applicability of this criterion depends on the form of the nonlinearity and on the form of trapping potential [36]. In the case of an attractive BEC confined in a magnetic trap, for example, a general stability criterion is derived for the ground states of the GP equation in Ref. [37]. This criterion states that a localized state is stable only if the chemical potential $\mu$ and the number of atoms $N=\int_{-\infty}^{\infty}|u(x)|^{2} d x$ satisfy $\partial N / \partial \mu<0$ [37]. The chemical potential $\mu$ corresponds to $-\Lambda$ of Ref. [37]. As for the repulsive nonlinearity, however, the stability of gap soliton families obeys an inverted ("anti-VK") criterion $\partial N / \partial \mu>0$ [38]. In Eq. (6), the number of atoms $N$ is linearly related to the nonlinearity via $g=2 a \mathrm{~N} / a_{\perp}^{2}$, and the wave function is normalized as $\int_{-\infty}^{\infty}|u(x)|^{2} d x=1$. Then, the anti-VK criterion can be expressed as $\partial g / \partial \mu>0$. Figures 3(b) and 3(d) show that the anti-VK criterion $\partial g / \partial \mu>0$ holds; hence the localized states are stable in the parameter range. To justify the anti-VK criterion, the stability of the localized BECs is tested in systematic numerical integration of GP equation (6) with $g>0$. For example, with $g=10$, first we create a localized BEC in a nonperiodic OL. Then, the stability of the localized states was established by slightly changing the potential (by multiplying by a factor of 0.98 ) in the real-time routine and continuing the time evolution to 10000 units of time. We find that the localized states remain localized in the real-time routine and hence are considered to be stable. At large time scales, the behavior of an initially localized wave packet has been analyzed numerically in Ref. [18]. It was shown that above a certain critical strength of nonlinearity a subdiffusion occurs. We emphasize here that, in our numerical calculations, the stationary localized states are obtained in an adiabatic way, and the stability of the localized states was tested within a short period of time.

Nevertheless, the anti-VK criterion cannot be naturally extended to the noninteracting BEC (viz., $g=0$ ). To test the stability of these noninteracting localized BEC, first we create a localized BEC in a nonperiodic OL. Successively, at $t=0$, we suddenly introduce a small initial momentum $p_{0}=0.1$ by changing $u(x) \rightarrow u_{0}(x) \exp \left(i p_{0} x\right)$, where $u_{0}(x)$ is the wave function of the localized BEC at $t=0$. The center $x_{0}$ of the localized states versus time $t$ is presented in Fig. 4, where $x_{0}$ is obtained by instant Gaussian-function fitting to the central region of the density distribution. It is found that after the perturbation, the localized states perform a sustained oscillations but remain localized, which implies that the localized states are stable against small perturbations. Contrary to our intuition, however, the oscillations are quasiperiodic because of the deformation of the density profile, as explained in Ref. [39].

One interesting earmark of Anderson localization in a weak disordered potential is a long exponential tail of the localized state in spite of the Gaussian distribution of the central part

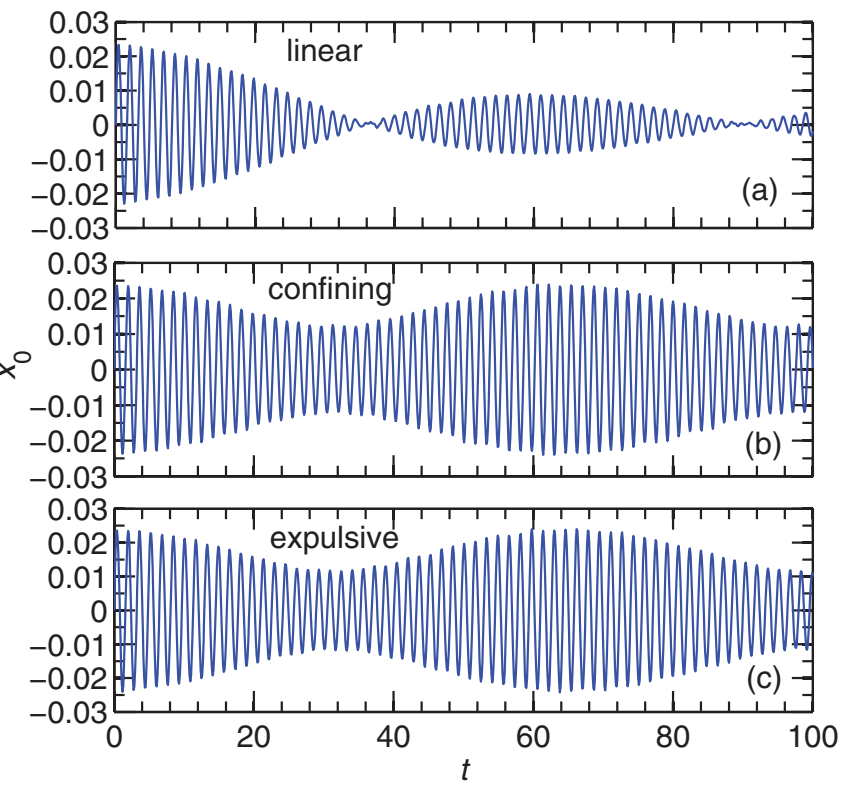

FIG. 4. (Color online) Center of the localized state $x_{0}$ vs time $t$ during the oscillation of the localized BEC on the nonperiodic OL (4) and (5) initiated suddenly by introducing an initial momentum $p_{0}=$ 0.1 through the transformation $u(x) \rightarrow u_{0}(x) \exp \left(i p_{0} x\right)$ with $g=0$ and $C=0.005$ for the (a) linear, (b) confining, and (c) expulsive perturbations.

[40]. To observe the effect of different perturbations of the $\mathrm{OL}$ on the tail of localized state, we present in Fig. 5 the density profiles of the localized BEC in log scale. As shown in Fig. 5(a), the localized BECs in the three perturbed OLs have long exponential tails extending far beyond the central Gaussian distribution. Further investigations show that the exponential tails are symmetric with respect to $x=0$ for the confining and expulsive perturbations, i.e., both left and right localization lengths are the same. In these cases, the
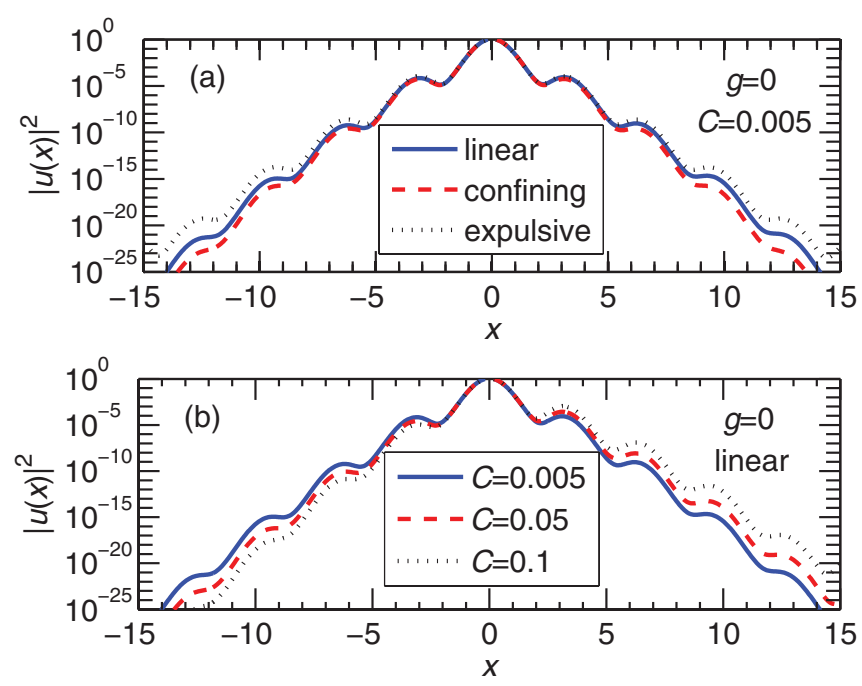

FIG. 5. (Color online) (a) Normalized numerical density profile $|u(x)|^{2}$ of the localized BEC vs $x$ with $g=0$ and $C=0.005$ for the linear, confining, and expulsive perturbations. (b) Normalized numerical density profile $|u(x)|^{2}$ of the localized BEC vs $x$ with $g=0$ and $C=0.005,0.05,0.1$ for the linear perturbations. 

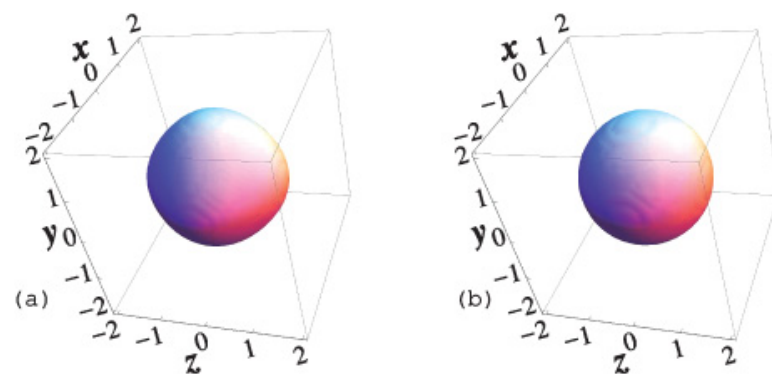

FIG. 6. (Color online) The 3D contour plot of normalized density profile of the localized state for $G=0$ from (a) numerical calculation and (b) variational approximation with the 3D OL perturbed by the expulsive potential (5) with $C=0.005$. In the plot the contour was set at a density of 0.001 .

localization length decreases with the increase of $C$. For linear perturbation, both the perturbed $\mathrm{OL}$ and the exponential tails are asymmetric with respect to $x=0$, and the asymmetry increases with the increase of the perturbation strength $C$, as shown in Fig. 5(b). Although the localization lengths depend on the perturbation strength $C$, the dependence is weak for small $C$.

Now we study localization in the 3D system with a perturbed OL [Eq. (3)]. As in a 1D system, the results for three types of perturbations are very similar in the 3D system. Hence, in a 3D system we illustrate the results for the expulsive perturbation only. First, we illustrate the results for the noninteracting case: $G=0$. To test the stability of the localized state for $G=0$ we use the real-time propagation of the 3D GP equation (1) with the initial state at $t=0$ taken as the variational localized state. The initial solution settles down to the final localized state after iteration during an adequate interval of time. The 3D contour plot of the density profiles of the localized state with its initial variational counterpart are shown in Figs. 6(a) and 6(b). Although the variational solution is spherically symmetric, this symmetry is absent in the actual localized state. This is because the $3 \mathrm{D}$ perturbed $\mathrm{OL}$ is not spherically symmetric. In spite of this, the variational approximation is very useful in the study of localization, and the numerical solution is nearly spherically symmetric. The variational chemical potential is -8.6826 compared to the numerical result of -8.690 .

Next, we study 3D localization in a repulsive system with $G=10$. Numerically, we find the stable localized state by real-time propagation with the variational approximation as the initial input. The 3D contour plots of the localized state with its variational counterpart are shown in Figs. 7(a) and 7(b). For $G=10$, the numerical profile of the localized state in Fig. 7(a) shows more deviation from spherical symmetry compared to Fig. 6(a) for $G=0$. The variational chemical potential of the localized state in Fig. 7(a) is -5.604 compared to the numerical result of -5.65 . In Fig. 7(c) we show the numerical chemical potential of the localized states for different $G$ and compare that with the variational results. Finally, we test the stability of the localized state with $G=10$. While obtaining the localized state, during real-time propagation we suddenly change $G$ from 10 to 11 , making the system more repulsive. Consequently, the root-mean-square size $w$ increases, and the system executes breathing oscillation. In Fig. 7(d) we plot
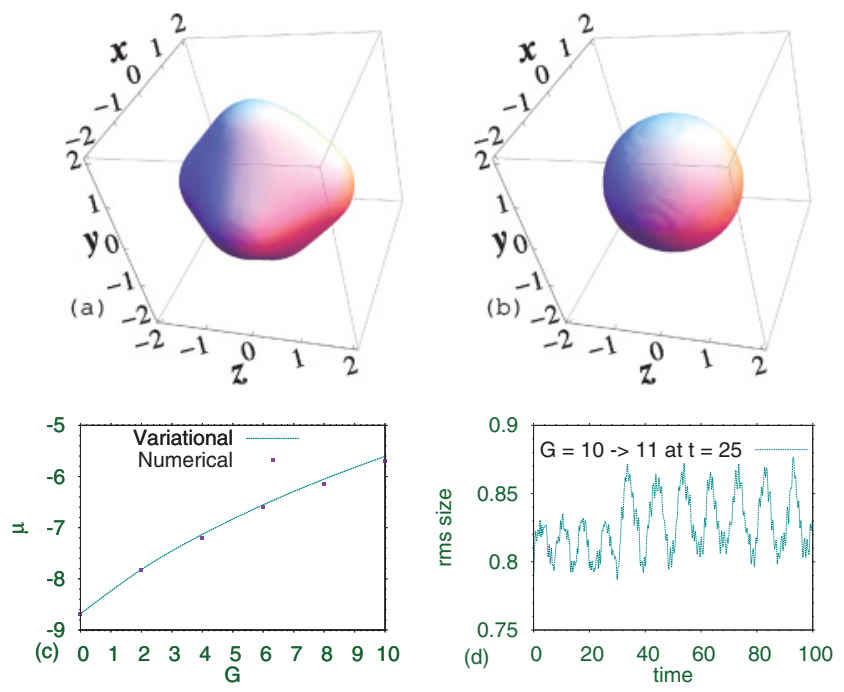

FIG. 7. (Color online) (a) and (b) The same as Fig. 6 for $G=10$. (c) Chemical potential $\mu$ for different $G$ from numerical simulation and variational approximation. (d) Width $w$ for $G=10$ during real-time evolution when at $t=25$ the nonlinearity $G$ was suddenly changed from 10 to 11 .

the root-mean-square size of the system during time evolution when $G$ was increased at $t=25$. The sustained oscillation of the system demonstrates the stability of the localized state. The variational equation (11) gives the largest value of $G$ for which there is a localized state. Real solutions for the width are allowed for $G<230.7$, beyond which there is no localization.

\section{SUMMARY AND DISCUSSION}

We suggested several simple schemes for removing the periodicity of an OL by adding a weak nonperiodic perturbation to study Anderson localization. We suggested three perturbations in the form of linear, confining harmonic, and expulsive potentials. All these perturbing potentials can be created in the laboratory, and such perturbed lattices can easily be used for BEC study. Usually, a bichromatic lattice is used in BEC studies, where the periodicity of the principal OL is removed by adding a secondary OL of incommensurate wave length.

Using the weakly perturbed OL we studied the problem of localization of a noninteracting or weakly interacting BEC in 1D and 3D systems using numerical and variational approaches to the GP equation. We studied the density profile and chemical potential of the localized states, where good agreement between variational and numerical results was established. The stability of the localized state was established by the Vakhitov-Kolokolov criterion and complemented by numerical studies of stable oscillation of the localized state upon small perturbation.

In this study we were mostly concerned with stationary localized states of BECs in one and three dimensions in nonperiodic potential and their stability. We are not concerned with the very long time dynamics of an arbitrary initial state in such a potential. For example, in a 1D weakly nonlinear disordered lattice Anderson localization is destroyed, and the field spreads subdiffusively far beyond the linear localization 
zone at large times [18]. Also, in three dimensions if a wave packet propagates in a linear disordered potential, at intermediate times there is a diffusion regime (due to atoms that have energies above the mobility edge), and at long times, if the disorder is strong enough, a remaining localized part (due to atoms that have energies below the mobility edge) will emerge [4,29]. Nevertheless, we demonstrate here using a mean-field model that stationary localized states are possible in a disordered potential in one and three dimensions for a relatively large nonlinearity or atomic interaction, and this could motivate experiments in the localization of BEC.

\section{ACKNOWLEDGMENTS}

FAPESP and CNPq (Brazil) provided partial support. Y. Cheng undertook this work with the Science and Technology Program of the Education Department of Hubei, China, under Grants No. D200722003 and No. Z200722001.
[1] P. W. Anderson, Phys. Rev. 109, 1492 (1958).

[2] J. Billy, V. Josse, Z. Zuo et al., Nature (London) 453, 891 (2008).

[3] G. Roati, C. D'Errico, L. Fallani et al., Nature (London) 453, 895 (2008).

[4] S. S. Kondov, W. R. McGehee, J. J. Zirbel, and B. DeMarco, Science 334, 66 (2011).

[5] F. Jendrzejewski, A. Bernard, K. Müller et al., e-print arXiv: $1108.0137 \mathrm{v} 2$.

[6] L. Fallani, J. E. Lye, V. Guarrera, C. Fort, and M. Inguscio, Phys. Rev. Lett. 98, 130404 (2007).

[7] A. Eckardt, M. Holthaus, H. Lignier, A. Zenesini, D. Ciampini, O. Morsch, and E. Arimondo, Phys. Rev. A 79, 013611 (2009).

[8] S. Ospelkaus, C. Ospelkaus, O. Wille, M. Succo, P. Ernst, K. Sengstock, and K. Bongs, Phys. Rev. Lett. 96, 180403 (2006).

[9] E. E. Edwards, M. Beeler, T. Hong, and S. L. Rolston, Phys. Rev. Lett. 101, 260402 (2008); C. Fort, L. Fallani, V. Guarrera, J. E. Lye, M. Modugno, D. S. Wiersma, and M. Inguscio, ibid. 95, 170410 (2005); B. Deissler, M. Zaccanti, and G. Roati et al., Nat. Phys. 6, 354 (2010).

[10] M. Larcher, F. Dalfovo, and M. Modugno, Phys. Rev. A 80, 053606 (2009).

[11] S. K. Adhikari and L. Salasnich, Phys. Rev. A 80, 023606 (2009); Y. Cheng and S. K. Adhikari, ibid. 81, 023620 (2010); S. K. Adhikari, ibid. 81, 043636 (2010); P. Muruganandam, R. K. Kumar, and S. K. Adhikari, J. Phys. B 43, 205305 (2010).

[12] Y. Cheng and S. K. Adhikari, Phys. Rev. A 84, 023632 (2011); Laser Phys. Lett. 7, 824 (2010); M. Modugno, New J. Phys. 11, 033023 (2009); D. J. Boers, B. Goedeke, D. Hinrichs, and M. Holthaus, Phys. Rev. A 75, 063404 (2007); G. Roux, T. Barthel, I. P. McCulloch, C. Kollath, U. Schollwock, and T. Giamarchi, ibid. 78, 023628 (2008); T. Roscilde, ibid. 77, 063605 (2008); X. Cai, S. Chen, and Y. Wang, ibid. 81, 023626 (2010); X. Deng, R. Citro, E. Orignac, and A. Minguzzi, Eur. Phys. J. B 68, 435 (2009); T. Paul, M. Albert, P. Schlagheck, P. Leboeuf, and N. Pavloff, Phys. Rev. A 80, 033615 (2009); S. Aubry and G. André, Ann. Isr. Phys. Soc. 3, 133 (1980); A. Iomin and S. Fishman, Phys. Rev. E 76, 056607 (2007).

[13] U. Gavish and Y. Castin, Phys. Rev. Lett. 95, 020401 (2005).

[14] P. Massignan and Y. Castin, Phys. Rev. A 74, 013616 (2006).

[15] L. Sanchez-Palencia, D. Clément, P. Lugan et al., New J. Phys. 10, 045019 (2008).

[16] N. Cherroret and S. E. Skipetrov, Phys. Rev. A 79, 063604 (2009); T. Nattermann and V. L. Pokrovsky, Phys. Rev. Lett. 100, 060402 (2008).

[17] Y. Cheng and S. K. Adhikari, Phys. Rev. A 82, 013631 (2010); G. Kopidakis, S. Komineas, S. Flach, and S. Aubry, Phys.
Rev. Lett. 100, 084103 (2008); I. García-Mata and D. L. Shepelyansky, Phys. Rev. E 79, 026205 (2009); Ch. Skokos, D. O. Krimer, S. Komineas, and S. Flach, ibid. 79, 056211 (2009).

[18] S. Flach, D. O. Krimer, and Ch. Skokos, Phys. Rev. Lett. 102, 024101 (2009); A. S. Pikovsky and D. L. Shepelyansky, ibid. 100, 094101 (2008).

[19] L. Sanchez-Palencia and L. Santos, Phys. Rev. A 72, 053607 (2005).

[20] B. Damski, J. Zakrzewski, L. Santos, P. Zoller, and M. Lewenstein, Phys. Rev. Lett. 91, 080403 (2003); T. Schulte, S. Drenkelforth, J. Kruse, W. Ertmer, J. Arlt, K. Sacha, J. Zakrzewski, and M. Lewenstein, ibid. 95, 170411 (2005).

[21] L. Sanchez-Palencia, D. Clément, P. Lugan, P. Bouyer, G. V. Shlyapnikov, and A. Aspect, Phys. Rev. Lett. 98, 210401 (2007); D. Clément, A. F. Varón, J. A. Retter et al., New J. Phys. 8, 165 (2006).

[22] J. E. Lye, L. Fallani, M. Modugno, D. S. Wiersma, C. Fort, and M. Inguscio, Phys. Rev. Lett. 95, 070401 (2005).

[23] D. Clément, A. F. Varón, M. Hugbart, J. A. Retter, P. Bouyer, L. Sanchez-Palencia, D. M. Gangardt, G. V. Shlyapnikov, and A. Aspect, Phys. Rev. Lett. 95, 170409 (2005).

[24] L. Fallani, C. Fort, and M. Inguscio, Adv. At. Mol. Opt. Phys. 56, 119 (2008).

[25] I. Bloch, Nat. Phys. 1, 23 (2005).

[26] A. del Campo and J. G. Muga, J. Phys. A 39, 5897 (2006); H. J. Metcalf and P. van der Straten, Laser Cooling and Trapping (Springer, New York, 1999); T. Esslinger, I. Bloch, and T. W. Hänsch, Phys. Rev. A 58, R2664 (1998); M. J. Ablowitz, K. Julien, Z. H. Musslimani, and M. I. Weinstein, Phys. Rev. E 71, 055602 (2005).

[27] B. P. Anderson and M. A. Kasevich, Science 282, 1686 (1998).

[28] L. Khaykovich, F. Schreck, G. Ferrari et al., Science 296, 1290 (2002).

[29] P. W. Anderson, Proc. Natl. Acad. Sci. USA 69, 1097 (1972); N. Mott, J. Phys. C 20, 3075 (1987).

[30] F. Dalfovo, S. Giorgini, L. P. Pitaevskii, and S. Stringari, Rev. Mod. Phys. 71, 463 (1999); A. J. Leggett, ibid. 73, 307 (2001).

[31] C. A. G. Buitrago and S. K. Adhikari, J. Phys. B 42, 215306 (2009); L. Salasnich, A. Parola, and L. Reatto, Phys. Rev. A 65, 043614 (2002).

[32] P. J. Y. Louis, E. A. Ostrovskaya, C. M. Savage, and Y. S. Kivshar, Phys. Rev. A 67, 013602 (2003).

[33] V. M. Pérez-García, H. Michinel, J. I. Cirac, M. Lewenstein, and P. Zoller, Phys. Rev. A 56, 1424 (1997); S. K. Adhikari and B. A. Malomed, ibid. 79, 015602 (2009); Y. Cheng, R. Z. Gong, 
and H. Li, Opt. Express 14, 3594 (2006); B. A. Malomed, Prog. Opt. 43, 69 (2002).

[34] P. Muruganandam and S. K. Adhikari, Comput. Phys. Commun. 180, 1888 (2009); S. K. Adhikari and P. Muruganandam, J. Phys. B 35, 2831 (2002).

[35] N. G. Vakhitov and A. A. Kolokolov, Izv. Vyssh. Uchebn. Zaved. Radiofiz. 16, 1020 (1973) [Radiophys. Quantum Electron. 16, 783 (1973)].
[36] L. Bergé, Phys. Rep. 303, 259 (1998).

[37] L. Bergé, T. J. Alexander, and Y. S. Kivshar, Phys. Rev. A 62 , 023607 (2000).

[38] H. Sakaguchi and B. A. Malomed, Phys. Rev. A 81, 013624 (2010).

[39] Y. Cheng and S. K. Adhikari, Phys. Rev. A 83, 023620 (2011).

[40] P. Bouyer, Rep. Prog. Phys. 73, 062401 (2010). 